\title{
Erratum to: Selection of Process Conditions for High Pressure Pasteurization of Sea Buckthorn Juice Retaining High Antioxidant Activity
}

\author{
Zacharias Alexandrakis • Konstantina Kyriakopoulou • \\ George Katsaros • Magdalini Krokida • Petros Taoukis
}

Published online: 23 July 2014

(C) Springer Science+Business Media New York 2014

\section{Erratum to: Food Bioprocess Technol}

DOI 10.1007/s11947-014-1299-5

The original version of the article unfortunately contains mistake. After the finalization of the proofs the authors have realized that they provided by mistake the wrong Acknowledgment for this work.

The acknowledgment is hereby corrected and presented.

Acknowledgments This research has been co-financed by the European Union (European Social Fund - ESF) and Greek national funds through the Operational Program "Education and Lifelong Learning" of the National Strategic Reference Framework (NSRF) - Research Funding Program: THALES: Reinforcement of the interdisciplinary and/or inter-institutional research and innovation.

The online version of the original article can be found at http://dx.doi.org/ 10.1007/s11947-014-1299-5.

\footnotetext{
Z. Alexandrakis $\cdot$ K. Kyriakopoulou $\cdot$ G. Katsaros $\cdot$

M. Krokida $(\triangle) \cdot$ P. Taoukis

School of Chemical Engineering, National Technical University of

Athens, Zografou Campus, 9 Iroon Polytechneiou, Athens 15780,

Greece

e-mail:mkrok@central.ntua.gr

Z. Alexandrakis

e-mail: zalexand@chemeng.ntua.gr

K. Kyriakopoulou

e-mail: kkyriakop@central.ntua.gr

G. Katsaros

e-mail: gkats@chemeng.ntua.gr

P. Taoukis

e-mail: taoukis@chemeng.ntua.gr
} 